\title{
Spatial prediction of landslide hazards in Hoa Binh province (Vietnam): a comparative assessment of the efficacy of evidential belief functions and fuzzy logic models
}

\begin{abstract}
The main objective of this study is to evaluate and compare the results of evidential belief functions and fuzzy logic models for spatial prediction of landslide hazards in the Hoa Binh province of Vietnam, using geographic information systems. First, a landslide inventory map showing the locations of 118 landslides that have occurred during the last ten years was constructed using data from various sources. Then, the landslide inventory was randomly partitioned into training and validation datasets $(70 \%$ of the known landslide locations were used for training and building the landslide models and the remaining $30 \%$ for the model validation). Secondly, nine landslide conditioning factors were selected (i.e., slope, aspect, relief amplitude, lithology, landuse, soil type, distance to roads, distance to rivers and distance to faults). Using these factors, landslide susceptibility index values were calculated using evidential belief functions and fuzzy logic models. Finally, landslide susceptibility maps were validated and compared using the validation dataset that was not used in the model building. The prediction-rate curves and area under the curves were calculated to assess prediction capability. The results show that all the models have good prediction capabilities. The model derived using evidential belief functions has the highest prediction capability. The model derived using fuzzy SUM has the lowest prediction capability. The fuzzy PRODUCT and fuzzy GAMMA models have almost the same prediction capabilities. In general, all the models yield reasonable results that may be used for preliminary landuse planning purposes.
\end{abstract}

Keyword: Landslide; GIS; Fuzzy operator; Evidential belief functions; Vietnam 